\title{
A progressive ground-glass opacity occupied the lower left lung in a postoperative colon cancer patient, what it will be? - a case report
}

\author{
Xiaodong Xie, Min Dong, Wenrong Shen \\ Departments of Radiology, Jiangsu Cancer Hospital, Jiangsu Institute of Cancer Research, Nanjing Medical University Affiliated Cancer Hospital, \\ Nanjing 210000, China \\ Correspondence to: Professor Wenrong Shen. Department of Radiology, Jiangsu Cancer Hospital, Jiangsu Institute of Cancer Research, Nanjing \\ Medical University Affiliated Cancer Hospital, 42 Baiziting, Nanjing 210000, China. Email: jszlyyct@126.com.
}

\begin{abstract}
Primary pulmonary mucosa-associated lymphoid tissue (MALT) lymphoma is a type of nonHodgkin lymphoma, and it occurs frequently in the stomach and rarely in the lungs. They are rarely taken into consideration in the differential diagnosis of lung lesions. MALT lymphomas affecting the lung show various styles on chest CT, which range from typical nodules or areas of consolidation to findings that are extremely rare, such as a progressive ground glass opacity (GGO) that never been reported before especially in a postoperative colon cancer patient. The postoperative pathologic diagnosis of the progressive GGO was extranodal marginal zone B-cell lymphoma of MALT. And during 2-year follow-up, the patient was in good condition and there was no sign of recurrence. This case would enrich the manifestations and increase the understanding in MALT, and inappropriate treatment would be avoided.
\end{abstract}

Keywords: Mucosa-associated lymphoid tissue lymphoma; ground glass opacity; lung; case report

Submitted Nov 15, 2019. Accepted for publication Dec 13, 2019.

doi: $10.21037 /$ tcr.2019.12.40

View this article at: http://dx.doi.org/10.21037/tcr.2019.12.40

\section{Introduction}

PPL is defined as a clonal lymphoid proliferation affecting one or both lungs in a patient with no detectable extrapulmonary involvement at the time of diagnosis or up to 3 months thereafter $(1,2)$. It is an uncommon neoplasm that represents only $0.4 \%$ of primary pulmonary malignancies $(3,4)$. The clinical symptoms and radiological features are often nonspecific, which may often misdiagnose as inflammation, tuberculosis, even lung cancer (5-8). It is significant for us to increase awareness of this rare disease, which will bring benefits to the therapeutic and prognostic implications. We herein report an unusual case of a localized pulmonary MALT lymphoma that showed as a progressive GGO in a woman patient with a 12 months history of colon cancer operation. The MALT lesion manifests as a progressive GGO has never been reported before, and the cancer history increased the difficulty and complexity of diagnosis. We present the following case in accordance with the CARE Guideline.

\section{Case presentation}

\section{Clinical diagnosis and treatment process}

A 56-year-old woman with a 12 months history of colon cancer operation (as shown in Figure 1), was found to have a few shadows with ground glass opacity in the lower left lung on chest CT in 2016. 07. 29 for the first time. At that time the density of the lesion was slight and with ground glass density, the shape was irregular and blurred, and we considered it to be inflammation. No evidence indicated that it was a neoplasm, therefore anti-inflammatory treatment was recommended. With the follow-up time from 2016-10-09 to 2017-06-20, totally 4 exams were done (as shown in Figure 2). The shadow in the lower left lung that was initially very small and slight increased in size and density over time, and presented as a typical 


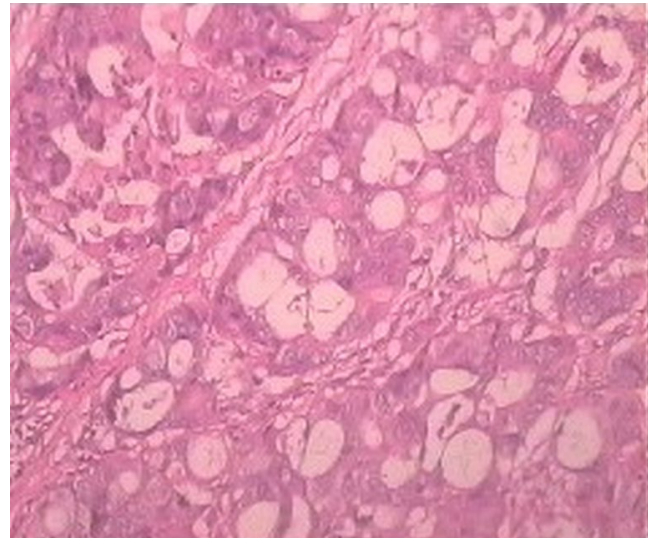

Figure 1 Colon cancer history. Infiltrating tubular-papillary adenocarcinoma, moderately-poorly differentiated, infiltrating whole layer of extrasolar adipose connective tissue. HE staining method $(\times 200)$. partial solid nodule with a size of $25 \mathrm{~mm} \times 20 \mathrm{~mm}$ before operation at 2017-06-20. Lung adenocarcinoma, metastasis, inflammation, or others? Anti-inflammation was useless and the lesion increased in size and density with passage of time, the suspicion of inflammation was eliminated first. The two specialized chest radiologists evaluated and analyzed all the images carefully, a consensus diagnosis that lung adenocarcinoma should be primary suspected was reached. Although, the history of colon cancer added the confusions to this lesion, they listed the metastasis as a second preference finally. Meantime, there was no newly lesions was detected in CT examination, the blood tests (routine blood, blood biochemistry, tumor marker, infectious disease indicators) were normal. The thoracic surgeon evaluated the systemic status of the patient with bowel cancer operation history, then a VATS was done.

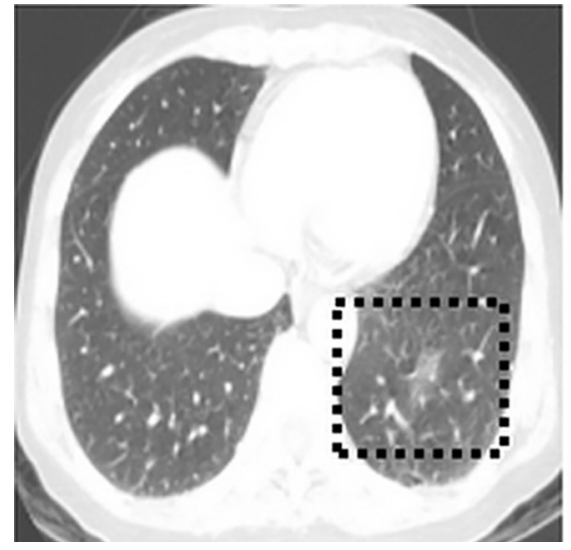

2016-07-29

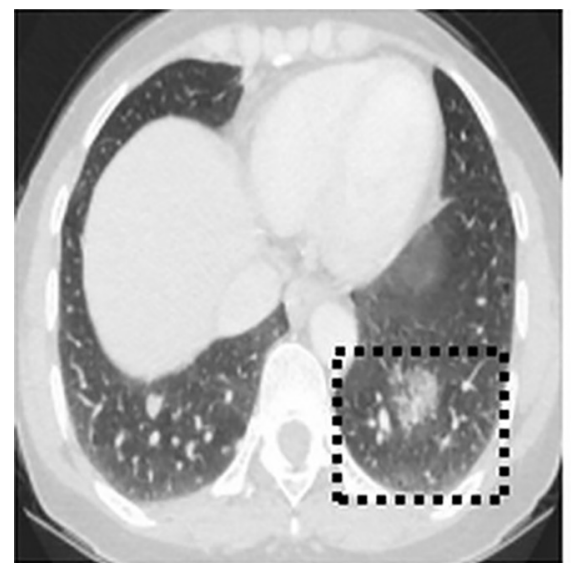

2017-06-20

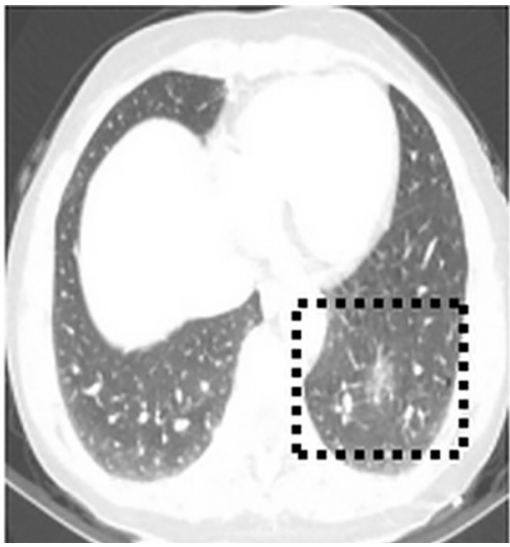

2016-10-09

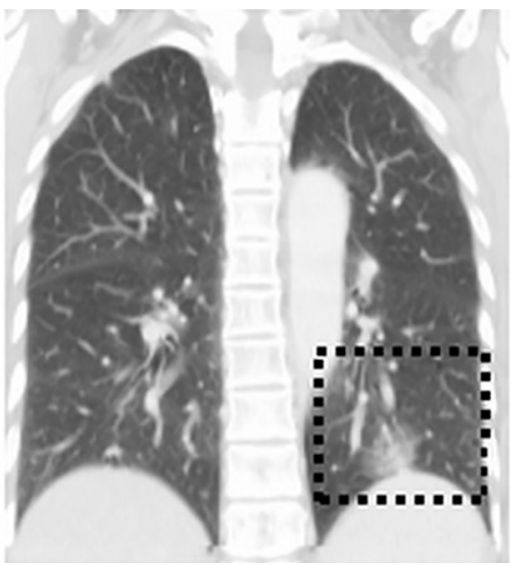

2017-06-20

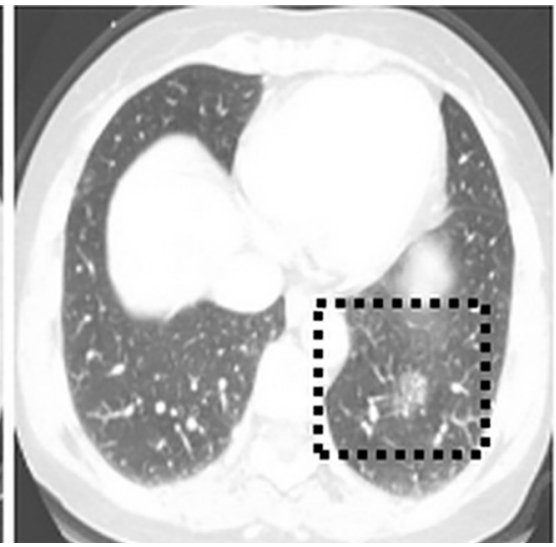

2016-12-30

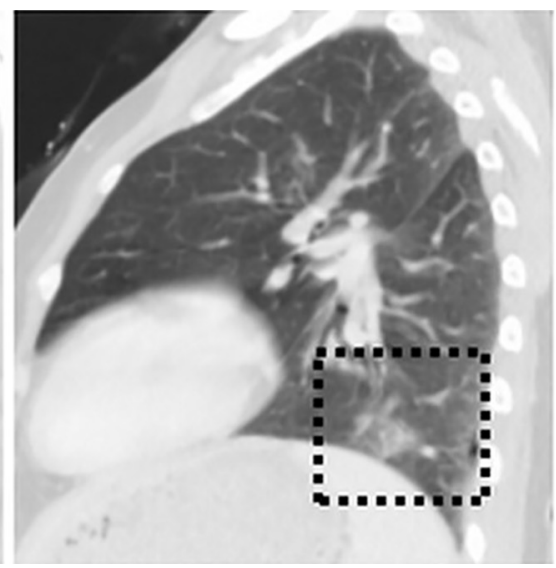

2017-06-20

Figure 2 The changes of the lesion in 4 different exams. The ground glass opacity in the lower left lung increased in size and density over time from 2016-07-29 to 2107-06-20, and presented as partial solid nodule with a size of $25 \mathrm{~mm} \times 20 \mathrm{~mm}$ in 2017.06.20. 


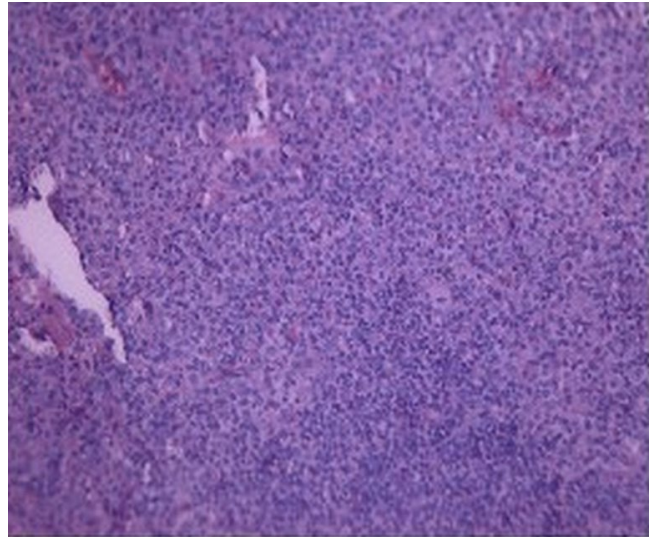

Figure 3 Pathological result. The lesion was proved to be a MALT. CD20(+), CD79a(+), CD3, CD5, CD43 interfollicular space(+), CD30(+), MUM-1(+), CD10, BCL-6 germinal center(+), Ki-67 germinal center $(+)$. Immunohistochemical staining method $(\times 100)$. MALT, mucosa-associated lymphoid tissue.

\section{Clinical result and follow-up}

Immunohistochemical staining (as shown in Figure 3) showed that tumor cells were positive for CD20(+), CD79a(+), CD3, CD5, CD43 interfollicular space(+), CD30(+), MUM-1(+), CD10, BCL-6 germinal center(+), $\mathrm{Ki}-67$ germinal center(+). The lesions were diagnosed as extranodal marginal zone B-cell lymphoma of MALT. Now within the 2-year follow-up, the patient actively cooperated with us remained well and there was no sign of recurrence.

\section{Discussion}

The nonspecific clinical symptoms and radiological appearance of MALT bring mounting troubles and difficulties in diagnosis and treatment $(6,8)$. In radiographic images, they can show as mass, node, patch or consolidate and mixed types, patients are often followed as a diagnosis of pneumonia, pulmonary tuberculosis, organizing pneumonia, or interstitial lung disease, even lung cancer. Therefor it is significant for us to increase awareness of this rare disease.

GGO is defined as a hazy opacity that preserves underlying bronchial and vascular margins on $\operatorname{HRCT}(9,10)$. Neoplastic GGO results mainly from alveolar wall covering tumor growth or hemorrhagic tumor $(11,12)$. Localized GGOs that persist for months have been considered to be the precancerous lesions such as atypical adenomatous hyperplasia and early-stage adenocarcinomas such as bronchioloalveolar carcinoma (13). Here, we reported a
MALT suffer with a history of colon cancer, whose CT features showed as a progressive GGO during the follow-up time, which is extremely rare.

PPL is equally common in men and women, with its incidence peaking during the 6th and 7 th decades of life $(4,14)$. The age of our patient is a litter younger than that of reported, the routine examination to colon cancer may account for the earlier finding. Although PPL has different clinical symptoms, including cough, fever, chest pain, apnea, weight loss, fatigue, night sweats, etc. They are nonspecific, which contribute little to the diagnosis. And at least onethird of the patients have no symptoms (4), in our case the patient feels well and without obvious symptoms. The blood investigations of our patient were all noncontributory, although anemia, thrombocytopenia, an elevated level of LDH and CPR as well as ESR were often detected in some other cases $(15,16)$. Whereas, we only reported one case of MALT here, lacking of persuasion to some extend, accurate data needed to be done with more cases.

The CT images of PPL are highly variable. There is a lower lobe predominance, presenting as patchy opacity or mass-like consolidation and multiple nodules in $50 \%$ of the cases $(4,17)$. Our patient owns a history of colon cancer, the lesion appeared as a progressive GGO, which is extremely rare and bring mounting troubles and more possibilities to the diagnosis. Lung adenocarcinoma, metastasis, inflammation, or others? Giving a systemic evaluation to all the images and treating with Anti-inflammatory method was ineffective, lung adenocarcinoma was primary suspected. There is almost no lung metastatic lesion presenting as a progressive GGO in colon cancer. However, considering a high malignant probability to this lesion and combining the history of colonic malignancy, we retained the diagnosis of metastasis. And finally, the pathological results gave us an amazing answer-MALT.

\section{Conclusions}

Without specific presentation, the diagnosis of MALT is quite challenging and often leads to misdiagnosis or delayed diagnosis. Our report of a progressive GGO in a colon cancer patient would enrich the knowledge and cognitive level in MALT area we considered.

\section{Acknowledgments}

Funding: None. 


\section{Footnote}

Conflicts of Interest: All authors have completed the ICMJE uniform disclosure form (available at http://dx.doi. org/10.21037/tcr.2019.12.40). The authors have no conflicts of interest to declare.

Ethical Statement: The authors are responsible for all aspects of the manuscript in ensuring that questions related to the integrity of any part of the work are appropriately investigated and resolved. All procedures performed in studies involving human participants were in accordance with the Declaration of Helsinki (as revised in 2013). Written informed consent was obtained from the patient for publication of this manuscript and any accompanying images.

Open Access Statement: This is an Open Access article distributed in accordance with the Creative Commons Attribution-NonCommercial-NoDerivs 4.0 International License (CC BY-NC-ND 4.0), which permits the noncommercial replication and distribution of the article with the strict proviso that no changes or edits are made and the original work is properly cited (including links to both the formal publication through the relevant DOI and the license). See: https://creativecommons.org/licenses/by-nc-nd/4.0/.

\section{References}

1. Cadranel J, Wislez M, Antoine M. Primary pulmonary lymphoma. Eur Respir J 2002;20:750-2.

2. Tanveer S, Damati A E, Baz A E, et al. Primary Pulmonary Hodgkin Lymphoma. Rare Tumors 2015;7:5968.

3. Wróbel T, Dzietczenia J, Prochorec-Sobieszek M, et al. Primary pulmonary diffuse large B-cell lymphoma. Am J Hematol 2012;87:107-8.

4. Parissis H. Forty years literature review of primary lung lymphoma. J Cardiothorac Surg 2011;6:23.

5. Catteeuw J, Koegelenberg CFN, Bruwer JW, et al. A 54-Year-Old Man Referred With Nonresolving Pneumonia. Chest 2014;146:e92-6.

Cite this article as: Xie X, Dong M, Shen W. A progressive ground-glass opacity occupied the lower left lung in a postoperative colon cancer patient, what it will be? - a case report. Transl Cancer Res 2020;9(3):2049-2052. doi: 10.21037/ tcr.2019.12.40
6. Tanriverdi E, Acat M, Ozgul G, et al. Primary pulmonary lymphoma: four different and unusual radiologic and clinical manifestations. Leuk Lymphoma 2017;58:1231-3.

7. Yoshino N, Hirata T, Takeuchi C, et al. Primary Pulmonary Mucosa-Associated Lymphoid Tissue Lymphoma with a Nodular Opacity: Report of a Case. J Nippon Med Sch 2017;84:87-9.

8. Yao D, Zhang L, Wu PL, et al. Clinical and misdiagnosed analysis of primary pulmonary lymphoma: a retrospective study. BMC Cancer 2018;18:281.

9. Austin JH, Müller NL, Friedman PJ, et al. Glossary of terms for CT of the lungs: recommendations of the Nomenclature Committee of the Fleischner Society. Radiology 1996;200:327-31.

10. Infante M, Lutman RF, Imparato S, et al. Differential diagnosis and management of focal ground-glass opacities. Eur Respir J 2009;33:821-7.

11. Primack SL, Hartman TE, Lee KS, et al. Pulmonary nodules and the CT halo sign. Radiology 1994;190:513-5.

12. Park CM, Goo JM, Lee HJ, et al. Nodular groundglass opacity at thin-section CT: histologic correlation and evaluation of change at follow-up. Radiographics 2007;27:391-408.

13. Hiramatsu $M$, Inagaki $T$, Inagaki $T$, et al. Pulmonary ground-glass opacity (GGO) lesions-large size and a history of lung cancer are risk factors for growth. J Thorac Oncol 2008;3:1245-50.

14. Majid N, Kamal e B, Oncology B, et al. Primary pulmonary lymphoma: About five cases and literature review. Lung India 2014;31:53-5.

15. Huang $\mathrm{H}$, Lu ZW, Jiang CG, et al. Clinical and prognostic characteristics of pulmonary mucosa-associated lymphoid tissue lymphoma: a retrospective analysis of 23 cases in a Chinese population. Chin Med J (Engl) 2011;124:1026-30.

16. Hu YH, Hsiao LT, Yang CF, et al. Prognostic factors of Chinese patients with primary pulmonary non-Hodgkin's lymphoma: the single-institute experience in Taiwan. Ann Hematol 2009;88:839-46.

17. Depew ZS, Vassallo R. Pulmonary mantle cell lymphoma: a rare manifestation of an uncommon condition. Rare Tumors 2012;4:e11. 